\title{
On the organization of stereotyped response sequences
}

\author{
BARRY SCHWARTZ \\ Swarthmore College, Swarthmore, Pennsylvania
}

\begin{abstract}
When pigeons are required to peck each of two keys in any order for reinforcement, stereotyped response sequences develop that are resistant to disruption by extinction, schedules of reinforcement, or contingencies requiring sequence variability. To test the hypothesis that stereotyped response sequences become integrated behavioral units, two experiments introduced within-sequence temporal delays of varying duration. Experiment 1 found that when a delay followed each peck in a sequence, there was substantial disruption of sequence performance that was independent of delay duration. However, such disruption was only temporary. Experiment 2 found that when the location of a delay within a sequence was varied, sequence disruption was a function of when, in a sequence, the delay occurred. Delays that occurred within sequence subunits had large effects, whereas delays that occurred between such subunits had small effects. The data indicate that pigeons can learn to bridge within-sequence delays, and suggest that response sequences are organized into "phrases."
\end{abstract}

Schwartz (1980, 1981a, 1981b, 1982a, 1982b; Schwartz \& Reilly, 1983, 1985) reported a series of experiments on the development and maintenance of a complex, sequential operant. These experiments reported procedures adapted from Vogel and Annau (1973). Pigeons were required to peck each of two response keys exactly four times, in any order. At the beginning of a trial, the top left light in a $5 \times 5$ matrix of lights was illuminated. Each peck on the left key moved the illuminated light across one position, and each peck on the right key moved it down one position. When the bottom right key was illuminated (four pecks on each key), reinforcement was delivered. A fifth peck on either key before a fourth on the other terminated the trial without reinforcement. Schwartz found, as had Vogel and Annau, that although 70 different successful sequences were possible, each pigeon developed one particular sequence that became dominant, sometimes occurring on more than $90 \%$ of all trials.

Given that such stereotyped sequences develop, a question arises as to whether the sequences become integrated behavioral units. One property that such units might possess is a resistance to being broken down in the face of environmental challenges. Schwartz (1981b) explored this possibility by exposing pigeons to extinction. He found that in pigeons with extensive training on the sequence task, extinction had almost no effect on sequence stereotypy. More specifically, given that animals responded at all in extinction, they produced the same dominant sequence as they had during reinforcement, with

This research was supported by NSF Grant BNS 82-06670, and by a Swarthmore College Faculty Research Grant. Reprint requests should be addressed to the author, Department of Psychology, Swarthmore College, Swarthmore, PA 19081. roughly the same relative frequency. In addition, the temporal properties of response sequences were not disrupted by extinction. Extinction certainly resulted in fewer responses per minute. However, when the temporal pattern of responding was analyzed into two components, latency (the time to begin a sequence from trial onset) and response time (the time to complete a sequence once it was begun), it turned out that although extinction produced marked increases in sequence latency, it had almost no effect on response time.

In a second experiment (Schwartz, 1982b), sequences were reinforced on fixed-interval and fixed-ratio reinforcement schedules, schedules whose effects on the temporal character of responding are well documented (Ferster \& Skinner, 1957). Virtually all characteristics of sequence responding retained their integrity on these schedules; sequence accuracy, stereotypy, and response time were roughly uniform throughout the fixed interval or fixed ratio. The temporal response patterning that is typically observed on these schedules was restricted to the latency to initiate response sequences, a property of sequences that is analogous to interresponse time as a property of individual responses.

In a third experiment (Schwartz \& Reilly, 1985), longterm retention of sequence performance was investigated. So long as experience that involved keypecking did not intervene, 60 -day intervals between sequence training and retention testing had virtually no effect on sequence performance.

Taken together, the results of these experiments provide a strong indication that stereotyped response sequences become integrated, functional behavioral units. The present experiments were concerned with exploring the nature of that integration. Perhaps the simplest manner in which sequences might be integrated is as response 
chains; each response in a sequence provides the stimulus that triggers the next response. On the basis of such a view, one might expect that the introduction of withinsequence, temporal delays would impair sequence performance by breaking up the response chains. Experiment 1 examined this possibility. On the other hand, sequence integration might involve more complex and differentiated organization than do simple response chains. Sequences might be organized in hierarchical fashion, with the entire unit decomposable into subunits which themselves have some sort of internal organization. There might be, in short, a "grammar" to sequences, with each sequence decomposable into "chunks," and each chunk, in turn, decomposable into smaller elements (e.g., Fountain, Henne, \& Hulse, 1984; Lashley, 1951; see Adams, 1984 , for a review of the literature on the learning of motor sequences). On the basis of this view, the amount of disruption caused by within-sequence temporal delays might depend on where in the sequence the delay occurred. Experiment 2 explored this possibility by introducing delays at different points within sequences.

\section{EXPERIMENT 1}

The possibility that response sequences are organized as response chains poses some difficulties. Consider a sequence that commonly emerges as a dominant one when exactly four pecks on each key are required for reinforcement: four left pecks followed by four right pecks. If these eight pecks are a simple response chain, with each response providing the stimulus to trigger the next, the pigeon must somehow be differentiating the first three pecks from the fourth. After each of the first three pecks, the pigeon pecks left; but after the fourth peck, the pigeon pecks right. It is not obvious what cue or cues the pigeon might be using to make this differentiation. A plausible explanation - that sequence organization involves not simply response-produced stimulation to keep the chain on track, but also matrix light position-response pairs-was ruled out in an earlier experiment (Schwartz, 1981a). In that experiment, Schwartz showed that efficient and stereotyped sequence performance did not depend on any specific relation between particular responses and particular matrix light positions. All that was required was that the matrix light position change systematically as responding within a sequence proceeded. Thus, the role of response-produced stimulation in sequence organization requires further exploration. If it is playing an important role, one would expect that the introduction of delays within sequences would impair sequence performance. A delay should have the effect of degrading the information provided by stimulation from the last response, with the result that response sequences should become less accurate and more variable. Experiment 1 tested this possibility by introducing delays of varying duration after each response within a sequence.

\section{Method}

Subjects. Eight White Carneaux pigeons that had served previously in a variety of experiments involving sequence performance were maintained at $80 \%$ of free-feeding weights.

Apparatus. Four Gerbrands G7313 pigeon chambers contained three-key pigeon intelligence panels. The keys were Gerbrands' normally closed keys, requiring a force of $1 \mathrm{~N}$ to operate. They were spaced $7.5 \mathrm{~cm}$ apart, center-to-center, and were located $21 \mathrm{~cm}$ above the grid floor. A grain hopper was located directly below the center key, $5.5 \mathrm{~cm}$ above the grid floor, and a pair of houselights were located in the ceiling of the chamber. The houselights were illuminated throughout experimental sessions, except during 4-sec feeder operations, when a light in the feeder was illuminated.

On the left side wall of each of the chambers was mounted a $5 \times 5$ matrix of red lights. The lights were $.84 \mathrm{~cm}$ in diameter, and $.04 \mathrm{~A}$ (Dialco No. 507-3917-1471-60D). The lights in the matrix were spaced $2 \mathrm{~cm}$ apart. The top row of lights was $20 \mathrm{~cm}$ from the grid floor, and the rightmost column (closest to the intelligence panel) was $4 \mathrm{~cm}$ from the panel.

Scheduling of experimental events, data collection, and data analysis were accomplished with a Digital Equipment Corporation PDP8/E digital computer using interfacing and software provided by State Systems Incorporated, Kalamazoo, Michigan.

Procedure. Because the pigeons had already had extensive experience with the sequence procedure, no pretraining was required. Each session consisted of 50 trials, separated by a 5 -sec intertrial interval. A trial began with the two side keys illuminated with white light and the top left matrix light illuminated. Each left keypeck moved the matrix light one position to the right, and each right keypeck moved the matrix light one position down. If the pigeon pecked each key exactly four times, in any order, moving the illuminated matrix light from the top left to the bottom right, reinforcement (4-sec access to grain) was delivered. Incorrect sequences terminated the trial without reinforcement.

After 40 sessions of exposure to this procedure, within-sequence delays were introduced. Each peck turned out the keylights and the matrix light for $0.5 \mathrm{sec}$, after which they were reilluminated. All other aspects of the procedure were unchanged. After 30 sessions with this delay, the pigeons experienced 20 sessions of the original procedure without a delay. This sequence of 30 sessions with a delay followed by 20 sessions without a delay was repeated six more times. Each time, the delay was different; the pigeons experienced delays of $1.0,2.0,4.0,2.0,1.0$, and $0.5 \mathrm{sec}$, in that order.

\section{Results and Discussion}

In past research, two principal measures of sequence performance have been reinforcements per session, a measure of sequence accuracy, and the frequency of each pigeon's dominant sequence, a measure of sequence stereotypy. These data are presented in Figures 1 and 2. The figures present group data for the last five sessions of exposure to each sequence procedure without a delay (indicated on the $\mathrm{X}$-axis as 0 delay), and for the first and last five sessions at each delay value.

Figure 1 presents data on reinforcements, or correct sequences, per session. With no delay, pigeons obtained between 40 and 43 reinforcers per 50 -trial session; sequence accuracy was quite stable throughout the course of the experiment. Each time a delay was introduced, there was a dramatic decrease in sequence accuracy, followed by a recovery over the course of exposure to the delay. Figure 2 presents data on the frequency of each pigeon's dominant sequence per session. With no delay, the 


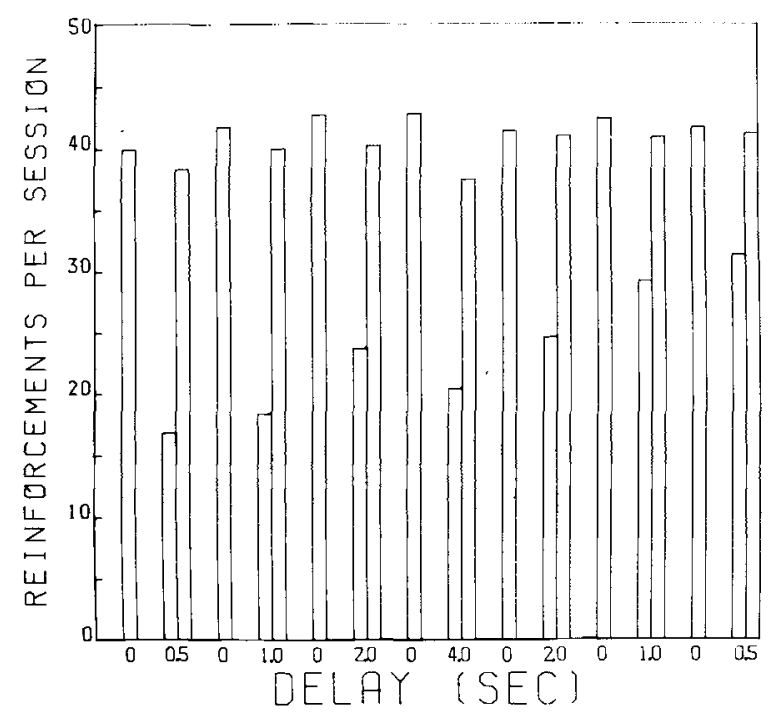

Figure 1. Reinforcements per session as a function of the delay imposed between pecks within sequences. Data are averaged across subjects for the last five sessions of each no-delay sequence procedure (single bars) and for the first and last five sessions of each sequence procedure with a delay (pairs of bars).

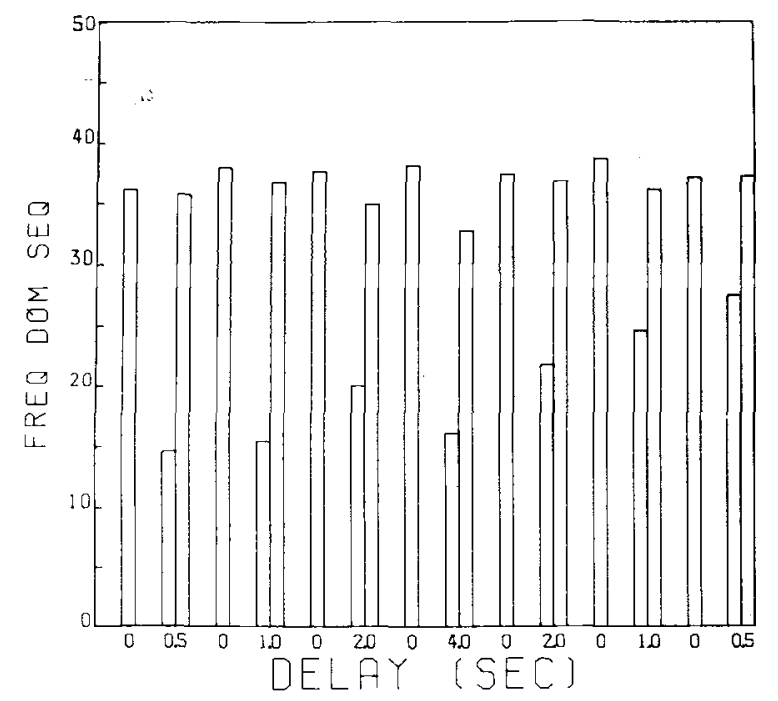

Figure 2. Frequency of the dominant sequence per session as a function of the delay imposed between pecks within sequences. Data are averaged across subjects for the last five sessions of each nodelay sequence procedure (single bars) and for the first and last five sessions of each sequence procedure with a delay (pairs of bars).

dominant sequence occurred on 35 to 38 of the 50 trials across all procedures. Introduction of delays produced a substantial decrease in the frequency of the dominant sequence, which recovered over the course of exposure to each delay. Thus, the introduction of delays produced a transient increase in sequence variability.

Statistical comparisons were made of asymptotic performance at each delay value and asymptotic performance when there was no delay. For the comparisons, data from all no-delay exposures were pooled, as were the data from pairs of exposures to delays of $0.5,1.0$, and $2.0 \mathrm{sec}$. Analyses of variance for delay were not significant for either of the two measures of sequence performance. Planned comparisons of each delay and the no-delay condition were then performed. The only significant comparisons were at the 4-sec delay; asymptotic reinforcements per session and frequency of the dominant sequence were significantly lower with the 4-sec delay than with no delay $[\mathrm{F}(1,28)=5.63 ; \mathrm{p}<.05]$.

To assess the disruption produced by the introduction of delays, difference scores were computed for each measure of sequence performance between the first five sessions of exposure to a given delay and the last five sessions of exposure to the no-delay procedure that had preceded it. An analysis of variance showed no reliable effect of delay magnitude on disruption of sequence performance. In addition, $t$ tests were performed to assess whether the difference scores were significantly greater than zero. At all delay values, $t$ tests were significant $(p<.01)$ for both reinforcements per session and frequency of the dominant sequence per session.

The present data indicate that the imposition of withinsequence delays disrupted sequence performance. However, the disruption was temporary. Over the course of exposure to each delay, recovery of sequence performance to baseline levels was virtually complete. The fact that delays produced sequence disruption suggests that response-produced stimulation plays some role in sequence organization (see Capaldi, Verry, Nawrocki, \& Miller, 1984, for similar conclusions derived from a quite different experimental context). One would expect such stimulation to decay over the course of a delay, with the result that sequence organization would suffer. On the other hand, the fact that recovery from the effects of delays was virtually complete suggests that pigeons developed, or always had available, some other source of information for use in sequence organization. The pigeons were observed in the delay procedures to see whether any differential patterns of behavior during the delays might have developed. Some of the pigeons did indeed develop reliable patterns of mediating behavior, especially at the 4-sec delay. In a few cases, they bobbed their heads in front of one of the keys. In two cases, they moved from side to side of the intelligence panel, with their breasts hugging the panel. But in no case did pigeons engage in mediating activities that differed as a function of where in the sequence they were. Thus, if the pigeons did develop some activity that helped bridge delays and keep sequences organized, it was not a peripheral activity.

\section{EXPERIMENT 2}

If sequences are organized as response chains, the disruptiveness of a within-sequence delay should be independent of where, within the sequence, it occurs. If, on the other hand, sequences are characterized by some sort of 
hierarchical organization, with the entire sequence decomposable into two or more subunits, which in turn may be decomposable into individual responses, then the location of a within-sequence delay may be critical. A delay that occurs within a subunit may be quite disruptive, as was the case in Experiment 1. However, a delay that occurs between subunits may not be disruptive at all. Indeed, it may even enhance performance, by sharpening the boundary between subunits. Such a result has been reported by Fountain et al. (1984) in a study of serial learning in rats. Rats were exposed to a regular pattern of reinforcement magnitudes, with repetition of the pattern separated by an interpattern interval. In some cases, the end of one pattern was set off from the beginning of the next by distinctive place or temporal cues. In other cases, these distinctive cues occurred within patterns. Rats learned the pattern only in the former cases. One might think of these cues as analogous to phrase markers; they enable the rat to chunk its long sequences of responses into subunits. When these markers occur at appropriate, "meaningful" places, pattern learning occurs; otherwise, it does not.

The present experiment attempted to explore whether the same phenomenon might be observed with pigeon response sequences. A single delay, of either 1 or $4 \mathrm{sec}$, was imposed after Response $1,3,4,5$, or 7 in a sequence. Of interest was whether the magnitude of sequence disruption was affected by where in a sequence the delay occurred. If we assume that sequence organization is characterized by some kind of hierarchical differentiation, what might we expect the effect of these different delay locations to be? To answer this question requires some speculation about what particular form hierarchical sequence organization might take, assuming that it is present at all. Such speculation is made easier by the fact that for most pigeons the dominant sequence is either four left pecks followed by four right pecks, or the reverse. For example, in Experiment 1, six of the eight pigeons had one of these two as the dominant sequence. Now intuition suggests that dominant sequences like these would be organized into two subunits, with the break between units coming at the point of switching between keys. If such subunits actually exist, we would expect that a delay after the fourth peck in a sequence would be much less disruptive than a delay at any other point, because only here would the delay occur between subunits. In addition, we might expect that delays after the first and third pecks would be more disruptive than delays after the fifth and seventh. The reasoning behind this is as follows: Suppose that sequence execution involves a series of "stay or switch" decisions together with the "rule" that once a switch occurs, the pigeon continues pecking the key it is on until the trial ends. If the pigeon is following a procedure like this, then behavior after the fourth peck and switch is quite mechanical and requires little or no organization. Delays after the fifth and seventh pecks occur after the pigeon has made its big "decision."

\section{Method}

Subjects. Eight White Carneaux pigeons, with previous experience on a variety of sequence procedures, were maintained at $80 \%$ of free-feeding weights.

Apparatus. The apparatus was the same as in Experiment 1.

Procedure. All procedural details were the same as in Experiment 1 , except that during procedures that involved within-sequence delays, only a single delay was introduced in each trial. All pigeons were exposed to delays of either 1- or 4-sec duration, after Response $1,3,4,5$, or 7 of each sequence. Each delay procedure was in effect for 3050 -trial sessions, and delay procedures were separated by 20 sessions of exposure to the sequence procedure with no delay. Four of the pigeons experienced all of the 4-sec delays first, and four of them experienced all of the 1-sec delays first. The order of delay location was different for each pigeon, and within pigeons, different for 1- and 4-sec delays.

\section{Results and Discussion}

The data of interest were the same as in Experiment 1: one measure of sequence accuracy (reinforcements per session), and one measure of sequence stereotypy (frequency per session of the dominant sequence). Preliminary analysis revealed that neither the magnitude of the delay ( 1 or $4 \mathrm{sec}$ ) nor the order of exposure to the two delays had any effect on sequence performance, either at asymptote or at points of transition. Thus, data from the two delay magnitudes were pooled. These data, averaged across subjects, are presented in Figures 3 and 4, which present the two measures of sequence performance as a function of the location, within sequences, of the delay.

Each figure presents data from the last five sessions of exposure to each sequence procedure with no delay, and from the first and last five sessions of each sequence procedure with a delay. As was generally the case in Ex-

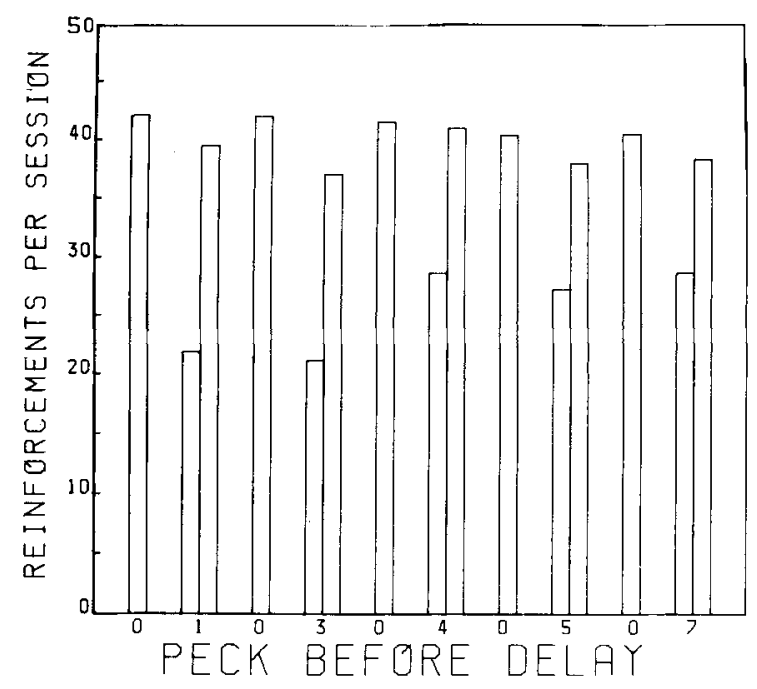

Figure 3. Reinforcements per session as a function of the location of the within-sequence delay. Data are averaged across subjects and across 1- and 4-sec delay values. Single bars present data from the last five sessions of no-delay procedures, and pairs of bars present data from the first and last five sessions of delay procedures. 


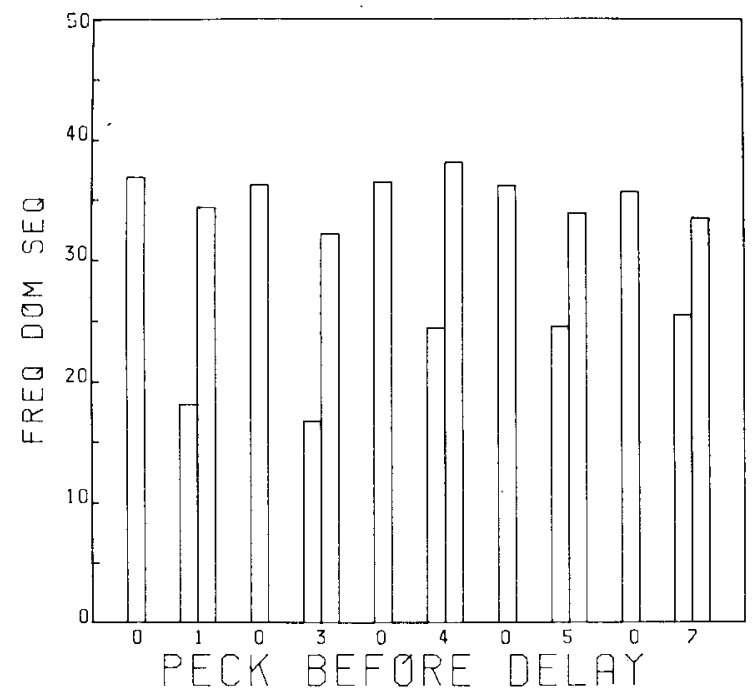

Figure 4. Frequency of the dominant sequence per session as a function of the location of the within-sequence delay. Data are averaged across subjects and across 1- and 4-sec delay values. Single bars present data from the last five sessions of no-delay procedures, and pairs of bars present data from the first and last five sessions of delay procedures.

periment 1 , there was no significant effect of delay location on any asymptotic measure of sequence performance. The pigeons obtained between 37 and 43 reinforcers per session, and produced their dominant sequences on between 32 and 38 trials per session. It is interesting to note, however, that sequence performance was more stereotyped at asymptote with a delay after the fourth response than it was even with no delay (Figure 4). This suggests that a delay at what was for most pigeons the major break in their sequences actually enhanced performance.

The differential effects of delay location are revealed when one looks at the amount of sequence disruption produced by the various delays when they were first introduced. As in Experiment 1, difference scores were computed between the first five sessions of exposure to each delay location and the last five sessions of the previous sequence procedure without a delay. An analysis of variance revealed a reliable effect of delay location on sequence disruption, in both accuracy $[\mathrm{F}(4,28)=3.12$, $\mathrm{p}<.05]$ and stereotypy $[\mathrm{F}(4,28)=3.65, \mathrm{p}<.05]$. In addition, planned comparisons were conducted to locate the sources of the effects. In both measures of sequence performance, the pattern was the same: Sequence disruption produced by delays after the first and third pecks was significantly greater than disruption produced by delays after the fourth, fifth, and seventh pecks ( $p<.05)$. The effects of Locations 1 and 3 were not different from each other, nor were the effects of Locations 4,5 , and 7 .

The effects of delay location can be seen more vividly in Figure 5, which presents difference scores pooled separately for all delays that occurred after the fourth peck in a sequence and all delays that occurred before it, at the 4-sec delay. The figure presents the mean decrease in reinforcements per session and in frequency of the dominant sequence per session in the first five sessions after the introduction of the delay. As is apparent from the figure, delay location had very substantial effects. Though disruption occurred at all delay locations, it was much larger when the delay occurred prior to the fourth peck than when it occurred after it. The same pattern of results obtained for the 1-sec delay.

It was indicated, in introducing this experiment, that if response sequences were organized into subunits, the precise composition of those units would likely depend on the form of the dominant sequence developed by each pigeon. In past research, dominant sequences have most often taken the form of four pecks on one of the keys followed by four pecks on the other. The same was true in this experiment. For five of the eight pigeons, the dominant sequence was either four left followed by four right pecks, or the reverse. For the other three pigeons, dominant sequences were as follows: LLRRRRLL, LRRRRLLL, and LLLRRRRL. If it is true that different delay locations have different effects not because of any absolute feature of location per se, but because of how delay location interacts with the organization of sequences, then the effects of delay location must be analyzed relative to the particular sequences that the delay is interrupting. There is, of course, no way to know from the form of a dominant sequence itself how that sequence is organized. It seems reasonable to assume, however, that if sequences are organized into subunits, breaks between subunits are much more likely to be located at points of switching between keys than at points within runs of pecks at a given key. Thus, it was assumed that for a sequence like LLLLRRRR, there are two subunits, with the break

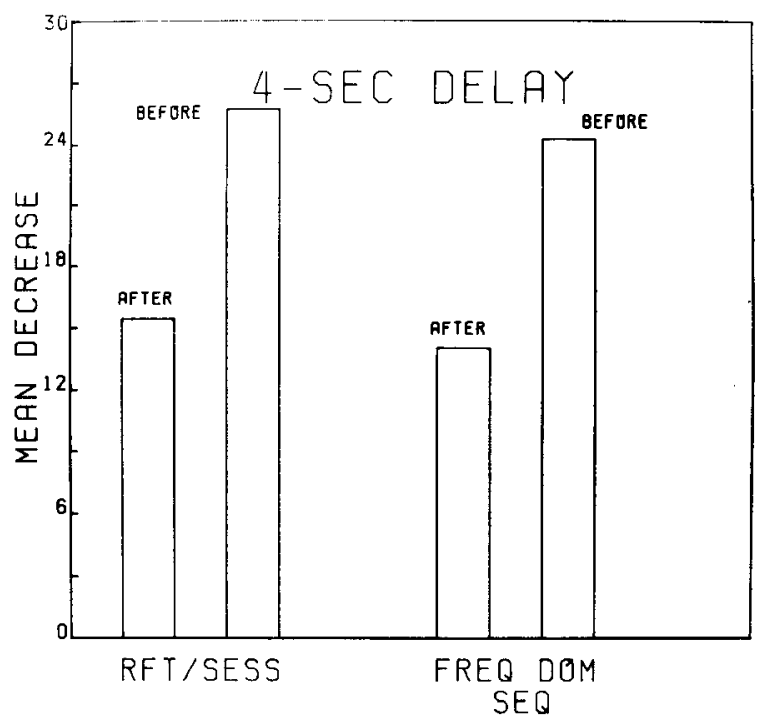

Figure 5. Mean decrease in reinforcements per session and frequency of the dominant sequence per session in the first five sessions of exposure to within-sequence delays as a function of whether the delays occurred before or after the fourth peck in a sequence. Data are averaged across all delay locations for the 4 -sec-delay value. 
between them coming after the fourth peck. On the other hand, for a sequence like LLRRRRLL, it was assumed that there are three subunits, with breaks coming after the second and sixth pecks. Under the guidance of this assumption about sequence organization, the effects of delay location were reanalyzed as a function of whether the delays had occurred at "major" or "minor" breaks in each pigeon's dominant sequence, with major breaks defined as points of switching between keys. Thus, for the five pigeons with the most common dominant sequence, the delay after the fourth peck would be viewed as coming at a major break, whereas the delays at all other locations would be viewed as coming at minor breaks. For the pigeon whose dominant sequence was LLRRRRLL, all delays would be viewed as coming at minor breaks. For the pigeon whose dominant sequence was LRRRRLLL, delays after Pecks 1 and 5 would be viewed as major and delays at other locations would be viewed as minor. Finally, for the pigeon whose dominant sequence was LLLRRRRL, delays after Pecks 3 and 7 would be viewed as major. The disruptive effects of delays located at all major breaks were combined and compared with the disruptive effects of delays located at all minor breaks. The expectation was that delays at major breaks woud be much less disruptive than delays at minor breaks. The results of this analysis are presented for the 1 -sec delay in Figure 6.

Figure 6 presents the decrease in reinforcements per session and frequency of the dominant sequence per session produced by the 1-sec delay as a function of whether its location was at a major or a minor break. The results are dramatic. Disruptions produced by delays at major

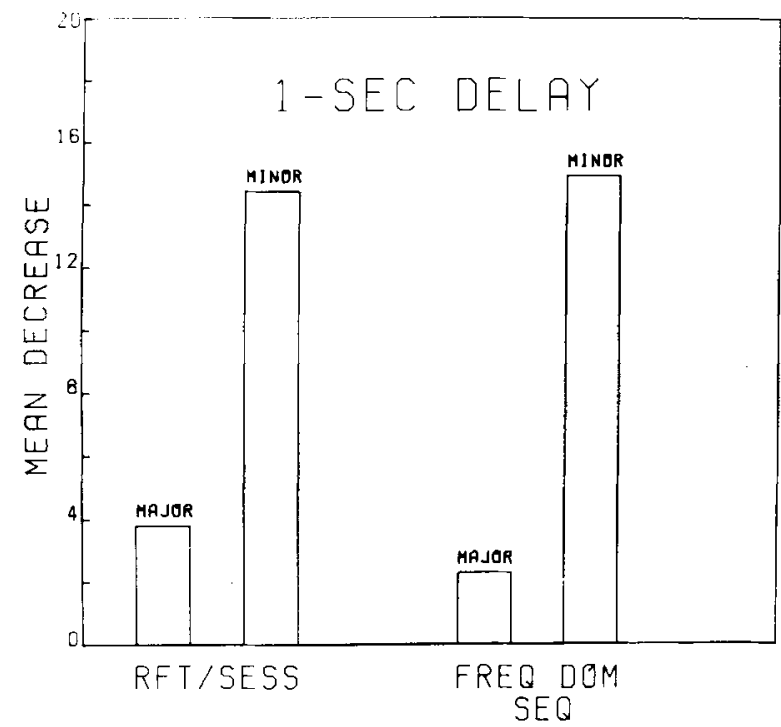

Figure 6. Mean decrease in reinforcements per session and frequency of the dominant sequence per session in the first five sessions of exposure to within-sequence delays as a function of whether the delays occurred at major or minor breaks in each pigeon's dominant sequence. Major breaks were defined as points of switching between keys, and minor breaks were defined as points within runs of pecks at a single key. Data are averaged across all delay locations for the 1-sec-delay value. breaks were quite small; indeed, they were not significantly different from zero. In contrast, the disruptions produced by delays at minor breaks were large and significantly different from zero $(p<, 01)$. Results for the 4-sec delay were similar but not identical. Here, the disruptive effects of delays even at major breaks were substantial, but the effects of major and minor breaks were still significantly different from each other $(p<.01)$.

The fact that where in a sequence a delay is located has such an important impact on its effects strongly suggests that sequences are organized not as undifferentiated response chains, but as "phrased" or hierarchical chunks. This result is consistent with other findings in the literature involving different species in different situations (Fountain et al., 1984). What this result adds to those other findings, however, is this: In the sequence task, no organization is dictated by the structure of the task itself. Dominant sequences can take a wide variety of different forms. Organization is, as it were, completely up to the pigeon. And it seems that with sequence organization largely unconstrained, pigeons establish behavioral units that are organized into hierarchical subunits.

There is another possible account of the effects of delay location that requires discussion. It is possible that there is a real difference between "programmed delay" and "effective delay" that is not constant across delay locations. It takes time for a pigeon to switch keys. This switching time amounts to an unprogrammed delay. Should the programmed delay coincide with the unprogrammed delay, then the effective programmed delay will be shorter. If, for example, a pigeon takes $0.5 \mathrm{sec}$ between pecks on the same key, but 2.0 sec between pecks on different keys, a 4-sec programmed delay will add $3.5 \mathrm{sec}$ in the former case but only $2.0 \mathrm{sec}$ in the latter. We have assumed that points of switching between keys constitute "major breaks" in sequences, but the relatively small disruption produced by delays at these breaks could be the result not of internal sequence organization, but of shorter effective delays. Though this account cannot be ruled out, several lines of evidence argue against it. First, both Experiment 1 and Experiment 2 showed little effect of delay magnitude per se. Second, pigeons typically execute an entire eight-peck sequence in less than $5 \mathrm{sec}$, and there is no clear effect on sequence execution time of the number of key switches in a pigeon's dominant sequence. Thus, it is unlikely that switching time is very lengthy. Finally, this account offers no explanation of the asymmetry between the effects of delays after Pecks 1 and 3 , and the effects of delays after Pecks 5 and 7 . For pigeons whose dominant sequence has a switch only after Peck 4, the effective delays before and after that switch should be the same. But the effects of these delays were not the same.

\section{GENERAL DISCUSSION}

As has now been demonstrated in a series of experiments, when reinforcement depends upon a sequence of 
responses that can be highly varied in form, stereotyped sequences develop. These stereotyped sequences are extremely resistant to disruption, and they are affected by manipulations of reinforcement conditions in much the same way that individual responses are. The present experiments introduced delays of varying durations at various points within sequences. The purpose of the experiments was twofold: first, to determine whether within-sequence delays would disrupt sequence performance, and second, to use delays to probe the nature of sequence organization.

With regard to the first objective, the results of the experiments are mixed. Within-sequence delays do disrupt sequence performance, but the disruption is temporary. When delays are first introduced, sequence accuracy decreases and sequence variability increases. But over the course of exposure to a given delay, performance recovers approximately to baseline levels. Results similar to these have been obtained in studies in which pigeons were required to produce sequences and perform a delayed matching-to-sample task concurrently (Schwartz \& Reilly, 1983). At asymptote, sequence performance was as accurate and stereotyped when concurrent matching was required as it was by itself. However, when matching was first introduced, or when the value of the delay between sample presentation and matching choice was changed, sequence performance underwent a transient deterioration. In discussing those results, Schwartz and Reilly suggested that when the matching task is introduced, and each time its parameters are changed, the pigeon is faced with a new problem, a new learning task. The task involves integrating the two separate tasks in a way that allows the pigeon to share its attention and processing resources between them in an efficient manner. The form this integration takes may well depend upon the specific details of the two tasks, so that each time one of the tasks is changed, a new mode of integration is required. The same sort of analysis may be appropriate to explain the significant, but transient, effects of within-sequence delays. It seems likely that delays are disruptive because they pose a challenge to sequence organization. Whatever the cues the pigeon depends upon to organize its sequences, they are sufficiently degraded by the delays for sequence organization to suffer. In response to this challenge, the pigeon comes to depend upon some other cues to organization that are less resistant to degradation by imposed delays. However, the effectiveness of the particular cues utilized may well be specific to the particular delay value imposed, so that a change in delay value creates a new organizational problem. It should be noted, incidentally, that the effects of delays in these experiments suggest that the perceptual cues provided by the light matrix are not essential to sequence organization (see also Schwartz, 1981a). To account for the different effects of delays after Pecks 1 and 3 and after Pecks 5 and 7, one would have to argue that some matrix cues are more salient or significant than others. Although an intuitive case could be made for the special salience of the bottom left or top right matrix cues, it is not obvious that a case could be made for the special salience of any of the others.

This analysis may not seem compelling, especially in the absence of any speculation about what the putative organizational cues might be. Consider, then, an alternative analysis, one based on the idea of generalization decrement. Perhaps the pigeon's performance is under the control of general contextual cues that inhere in the overall procedure. Any change in procedure alters these cues and thus disrupts performance. Performance is then reestablished, over time, under the control of the new contextual cues. Although this analysis may initially seem more plausible than the preceding one, there are two aspects of the present results that make it unlikely. First, one might expect that once a pigeon has adjusted to the contextual cues that inhere in, for example, a procedure with a 1-sec delay, a change to the no-delay procedure should change the context and disrupt performance. Although the data are not presented in any of the figures, shifts from delay to no-delay procedures never produced any disruption at all. Performance in the first five sessions of the no-delay procedure was the same in all respects as it was in the last five sessions. Second, there is no a priori reason to suppose that delays at some within-sequence locations should produce more generalization decrement than delays at other sequence locations. Yet, Experiment 2 clearly showed that the disruptiveness of a delay depends on where in a sequence it occurs. It seems, therefore, that a generalization-decrement account of these experiments will not do the job.

With respect to the second objective of these studies, it seems clear from the second experiment that response sequences are organized into subunits, with subunits marked by switches between keys. Delays within subunits produce substantial sequence disruption, whereas delays between them produce little disruption. If sequences were undifferentiated response chains, there would be no reason to expect that delays at some locations within a sequence would be more disruptive than delays at other locations. This conclusion is consistent with the results of quite different sequential learning tasks that have been studied with rats (e.g., Fountain \& Annau, 1984; Fountain et al., 1984; Hulse, 1978; Hulse \& Dorsky, 1977, 1979). The present experiments add to these by showing that even when sequence organization is spontaneous, that is, even when no particular organization is called forth by the structure of the task itself, the organization that emerges is phrased and hierarchical rather than undifferentiated and linear. If nonverbal behavior may be said to have a grammar, its general form, as noted by Lashley (1951) years ago, seems to have much in common with the grammar of verbal behavior.

\section{REFERENCES}

Adams, J. A. (1984). Learning of movement sequences. Psychological Bulletin, 96, 3-28.

Capaldi, E. J., Verry, D. J., Nawrocki, T. M., \& Miller, D. J. (1984). Serial learning, interitem associations, phrasing cues, inter- 
ference, overshadowing, chunking, memory, and extinction. Animal Learning \& Behavior, 12, 7-20.

FERSTER, C. B., \& SKINNER, B. F. (1957). Schedules of reinforcement. New York: Appleton-Century-Crofts.

Fountain, S. B., \& ANNAU, Z. (1984). Chunking, sorting, and rulelearning from serial patterns of brain stimulation reward by rats. Animal Learning \& Behavior, 12, $265-274$.

Fountain, S. B., Henne, D. R., \& Hulse, S. H. (1984). Phrasing cues and hierarchical organization in serial pattern learning by rats. Journal of Experimental Psychology: Animal Behavior Processes, 10, 30-45.

HulSE, S. H. (1978). Cognitive structure and serial pattern learning by animals. In S. H. Hulse, H. Fowler, \& W. K. Honig (Eds.), Cognitive processes in animal behavior (pp. 311-340). Hillsdale, NJ: Erlbaum.

Hulse, S. H., \& DoRSKY, N. P. (1977). Structural complexity as a determinant of serial pattern learning. Learning \& Motivation, 8 , 488-506.

HuLSE, S. H., \& DoRSKY, N. P. (1979). Serial pattern learning by rats: Transfer of a formally defined stimulus relationship and the significance of nonreinforcement. Animal Learning \& Behavior, 7, 211-220.

LASHLEY, K. (1951). The problem of serial order in behavior. In L. A. Jeffries (Ed.), Cerebral mechanisms in behavior (pp. 112-146). New York: Wiley.

SchwaRTz, B. (1980). Development of complex, stereotyped behavior in pigeons. Journal of the Experimental Analysis of Behavior, 33, 153-166.

SCHWARTz, B. (1981a). Control of complex, sequential operants by systematic visual information in pigeons. Journal of Experimental Psychology: Animal Behavior Processes, 7, 31-44.

SCHWARTZ, B. (1981b). Reinforcement creates behavioral units. Behaviour Analysis Letters, 1, 33-41.

SCHWARTZ, B. (1982a). Failure to produce response variability with reinforcement. Journal of the Experimental Analysis of Behavior, 37, 171-181.

SchWARTZ, B. (1982b). Interval and ratio reinforcement of a complex sequential operant in pigeons. Journal of the Experimental Analysis of Behavior, 37, 349-357.

SCHWARTZ, B., \& REILLY, M. (1983). Response stereotypy without automaticity in pigeons. Learning \& Motivation, 14, 253-270.

SChWARTZ, B., \& Reilly, M. (1985). Long term retention of a complex operant in pigeons. Journal of Experimental Psychology: Animal Behavior Processes, 11, 337-355.

VoGEl, R., ANNAU, Z. (1973). An operant discrimination task allowing variability of response patterning. Journal of the Experimental Analysis of Behavior, 20, 1-6.

(Manuscript received October 31, 1984; revision accepted for publication August 28, 1985.) 